\title{
Especialización y Diversificación de las exportaciones por países (1981 -2015)
}

\section{Export specialization and diversification by countries (1981 -2015)}

\author{
Katya Vanessa Macías Badaraco ${ }^{1 *}$, Evelyn Karina Tinoco Diaz ${ }^{1,2}$ y Jorge Luis Puyol Cortez $^{2}$ \\ ${ }^{1}$ Universidad Técnica Luis Vargas Torres, Extensión La Concordia \\ 2 Universidad Regional Autónoma de los Andes, Extensión "Santo Domingo" \\ *katya.macias.badaraco@gmail.com
}

DOI: https://doi.org/10.26871/killkana_social.v2i3.350

\begin{abstract}
Resumen
Las exportaciones de un país representan un sinnúmero de beneficios, tales como el ingreso de divisas, creación de fuentes de empleo, la reducción de costos, participación internacional, entre otros. No obstante, estas también lo exponen a ciertas vulnerabilidades. Así, el objetivo de este trabajo ha sido cuantificar el nivel de vulnerabilidad que enfrentan los países en el comercio exterior mediante el índice Herfindahl. Un valor elevado del índice se traduce en una gran concentración de las exportaciones en un sector determinado, y mayor vulnerabilidad ante cambios en la demanda sectorial. Es decir, que, a mayor diversificación exportadora, mayor es la capacidad de un país para hacer frente a las fluctuaciones económicas a nivel internacional. Por el contrario, a menor diversificación el país se encuentra en una situación de enorme fragilidad económica. La metodología utilizada fue de alcance descriptiva y cuantitativa. Específicamente, se analizaron los datos de la Organización Mundial de Comercio sobre las exportaciones anuales por sectores comerciales de 181 países, en el periodo 1981-2015. Con datos de panel se analizaron los factores determinantes del índice de concentración o vulnerabilidad, calculado para medir el grado de especialización o concentración de las exportaciones nacionales para cada país de la muestra. Las variables analizadas fueron: población, PIB, PIB per cápita, nivel de exportaciones en base al PIB y área geográfica. Los resultados muestran a Holanda, EE. UU. y Corea del Sur con el índice de especialización bajos. Mientras que, Irak, Groenlandia y Angola con altos. Se concluye que la diversificación maximiza la oferta exportable y reduce el impacto ante fluctuaciones internacionales.
\end{abstract}

Palabras clave: Comercio exterior, concentración, Índice Herfindahl.

\begin{abstract}
Exports bring a lot of benefits to a country, such as foreign exchange earnings, labor creation, cost reduction, and international participation. Nevertheless, exports also expose the country to some risks. The goal of this research work is to quantify the vulnerability level of countries involved in foreign trade through the Herfindhal index. The higher this index is, the higher the concentration of exports in one specific sector, which translates into greater vulnerability to changes or shifts in the sector's demand. Therefore, with more diversified exports, the ability of a country to face international economic fluctuations increases. Conversely, countries with poorly diversified exports are in a remarkably fragile economic condition. The methodology followed is both descriptive and quantitative. Data from the World Trade Organisation on annual exports per sector in 181 countries in the period 1987-2015 was analysed. The determining factors of the concentration or vulnerability index were analysed through panel data. This index is used to measure the exports specialisation or concentration level in the countries within the sample. The variables analysed are: population, GDP, GDP per capita, exports as \% of GDP, and exports per area. The results show that The Netherlands, U.S.A., and South Korea have the lowest specialisation index, and Iraq, Greenland and Angola the highest. The conclusion is that diversification maximises exports supply and reduces the impact of international fluctuations.
\end{abstract}

Key words: concentration, Herfindahl index, foreign trade, vulnerability.

\section{Introducción}

La globalización ha permitido en los últimos años que el acceso a líneas de comunicación y transporte facilite las formas de realizar negocios y mejorar el comercio entre países. Esto, a su vez, ha llevado a las autoridades a plantear políticas de desarrollo, establecer acuerdos comerciales y a formar bloques de integración económica creando mayores oportunidades para acercar productos hacia mercados cada vez más complejos, abriendo puertas a la diversificación.

Existen varios tipos de índice que miden la evolución de exportaciones de un país, los cuales ponen en relieve cierta capacidad o vulnerabilidad que tienen en el comercio 
internacional: los índices cuantitativos miden la concentración más que la diversificación (Carrère, Cadot, y StraussKahn, 2011), el índice de especialización mide el grado de conocimiento y tecnología impuesta en un sector de exportación específico. La vulnerabilidad en las exportaciones de cada país se encuentra medida principalmente por el volumen y la diversificación de productos en los distintos sectores exportadores. Los países desarrollados como Alemania y Japón han podido diversificar sus sectores y lograr volúmenes de exportaciones cada vez más altos convirtiéndose en países menos vulnerables que aquellos que se encuentran en vías de desarrollo.

Lograr crecimientos en los niveles de exportación para un país implica varios componentes que se deben analizar como ventajas comparativas, acceso a tecnologías, bloques comerciales, políticas administrativas locales, formas de financiación, ubicación geográfica, dotación de factores de producción o capital humano entre otros. En cada país estos elementos tienen un impacto de mayor o menor medida.

Los datos de las exportaciones mundiales por países y sectores comerciales han sido extraídos de la base de datos de la Organización Mundial de Comercio, correspondiente al período 1981-2015, dichos datos fueron procesados y analizados con el fin de alcanzar los siguientes objetivos: primero, analizar la información estadística de exportaciones por sectores a nivel mundial. Segundo, elaborar un índice de especialización de las exportaciones por países y sectores, basado en el cálculo del Índice Herfindahl. Luego, realizar con análisis clúster una clasificación de grupos de países de acuerdo con su capacidad o vulnerabilidad en el comercio exterior. Y, finalmente, realizar un análisis de las causas geográficas y económicas del índice de especialización de las exportaciones.

La importancia de analizar las exportaciones es medir el grado de especialización en ciertos sectores comerciales de cada país a nivel mundial y como esa concentración puede repercutir en su crecimiento económico o en la capacidad para hacer frente a fluctuaciones de los precios en el mercado internacional; por tanto, este trabajo es útil tanto para profesionales de las áreas de economía dado que crea un panorama de comercio exterior que facilita la toma de decisiones, como para el campo académico demostrando como la diversificación exportadora aumenta la resistencia ante shocks externos.

\subsection{Diversificación de las exportaciones}

La diversificación exportadora es un determinante importante de la estructura de las economías industriales modernas y tiene significativas implicaciones para la competencia y la eficiencia, como lo sugiere Cosh (1987) cuando define la diversificación como el proceso mediante el cual una corporación moderna extiende sus actividades más allá de los productos y mercados en los que opera actualmente. Es decir, la diversificación alude que las exportaciones están siendo sofisticadas, por tanto, las empresas se encuentran en la constante búsqueda por ofertar productos cada vez más desafiantes, tales nuevos productos involucran insumos muy específicos como conocimiento, activos físicos, capacitación laboral, necesidades de infraestructura, derechos de propiedad, requisitos reglamentarios y otros bienes públicos; Hausmann \& Klinger (2006) llaman a esto un cambio estructural por lo que junto al crecimiento dependen de los tipos de productos con los que cada país comercializa.

Si una economía depende en gran medida de las exportaciones de las materias primas o de los productos parcialmente terminados, es mucho más probable que se vea afectada por variaciones en los precios mundiales y habría poco por hacer para evitar una disminución en la actividad comercial o incluso una crisis. (Pacek y Thorniley, 2008)

Según los planteamientos de Adam Smith sobre el crecimiento económico (división del trabajo y especialización) y el modelo de comercio internacional de Heckscher-OhlinSamuelson (HOS), los países deben aprovechar sus ventajas comparativas para especializarse en la producción de determinados bienes, aunque recientes estudios han demostrado, por el contrario, que los países diversifican su producción y exportaciones a medida que crecen.

Para los países en desarrollo, la diversificación de exportaciones debe ser un factor clave a tomar en cuenta para el crecimiento económico, hacer frente a las fluctuaciones en los tipos de cambio, dar solución a la enfermedad holandesa, y para no depender únicamente de la producción de materias primas; así lo sugiere la Tesis de PrebischSinger (Singer, 1998).

De hecho, han existido varios estudios empíricos que resaltan que la diversificación contribuye a un mayor crecimiento económico (Chenery, Robinson, y Syrquin, 1986)(Syrquin, 1989). Además, en este mismo sentido, otras investigaciones afirman que los países deberían transitar de las exportaciones primarias a las exportaciones de manufacturas para lograr un crecimiento sostenible. (Hesse, 2009).

\subsection{Medición de Especialización}

Todos los índices de especialización generalmente miden la desigualdad entre las cuotas de exportación. La medida estadística más utilizada para medir la concentración es el índice Herfindahl (llamado también Índice de Hirschman-Herfindahl, HHI, en honor al científico Albert Hirschman quien lo puso en uso junto a Orris Herfindahl) (Agosin, 2009).

Con la elaboración de este índice se mide la especialización/diversificación de cada país en sus exportaciones; el HHI se define como:

$$
H H I_{j}=\sum i\left(X_{i j} / X_{T j}\right)^{2}
$$

donde $\mathrm{Xij}$ son las exportaciones del sector realizadas por el país j y XTj son las exportaciones totales del país j. Un valor del índice igual a 1 indica que la región se encuentra completamente especializada en un único sector, mientras que si toma valores cercanos a $1 / n$ (siendo $n$ el 
Tabla 1 Análisis del índice Hirschman-Herfindahl correspondiente a cada grupo de países por sectores exportadores (2015)

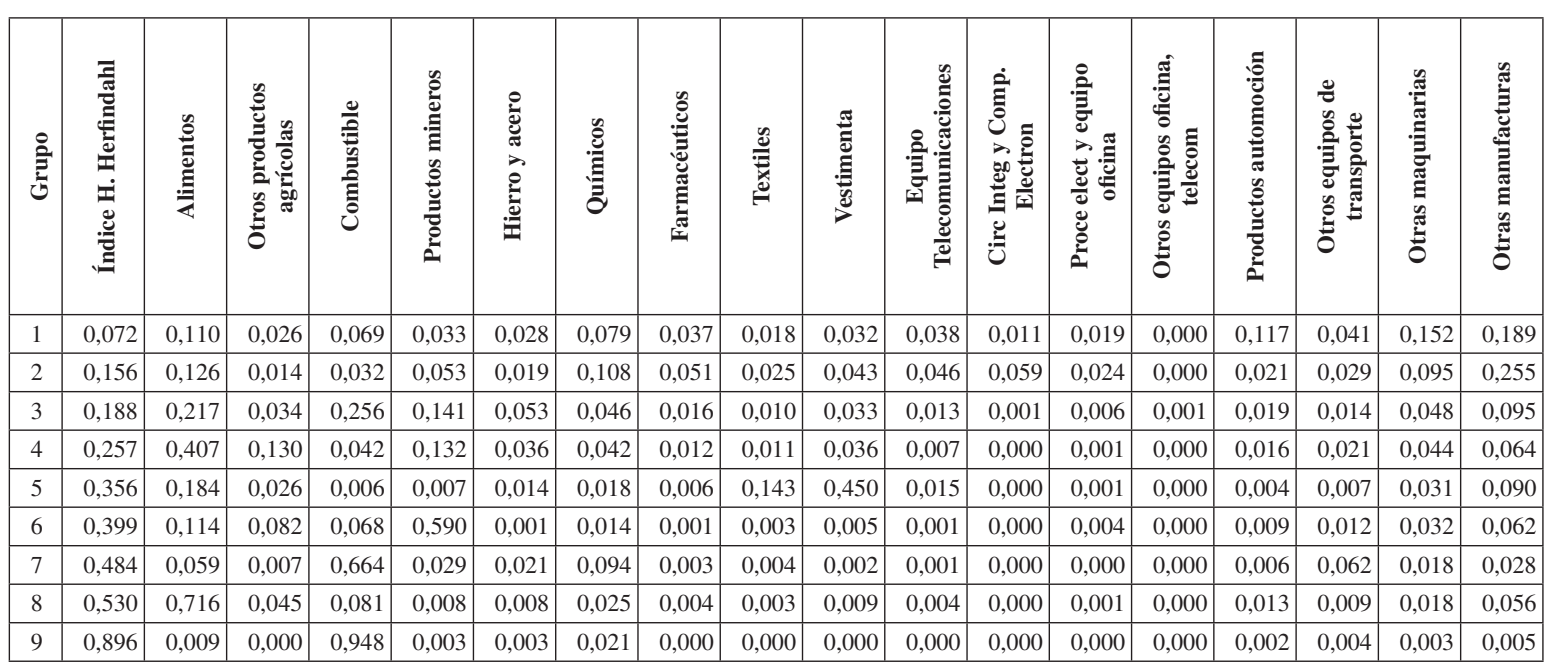

Fuente: Organización Mundial del Comercio

Elaborado por: Los autores

número de sectores considerados) implica que las exportaciones del país analizado se encuentran muy diversificadas entre los distintos sectores (Expósito, 2003). Para hacer que el valor mínimo del índice no dependa del número de sectores considerados se lleva a cabo una transformación por la que se le resta al índice HHI el inverso del número de sectores y se le divide entre uno menos el inverso del número de sectores. De esta forma los datos de distintos periodos se vuelven comparables.

1.3 Análisis de clústeres según la especialización y la importancia relativa de los sectores productivos en las exportaciones

Mediante el análisis clúster se han clasificado los países en nueve grupos, dicho número de clúster hace muy reducida la varianza entre grupos. Se opta por ese número de clústeres dado que la varianza entre grupos permanece casi constante con un mayor número. De este modo se consigue una clasificación lo suficientemente amplia de modo que los países cuyos perfiles son muy similares se han aglomerado tomando en cuenta el valor del HHI, el porcentaje exportado en cada sector comercial (17 sectores económicos).

En la Tabla 1 se observa el índice de Herfindahl promedio de cada conjunto de países correspondiente al año 2015, y la participación porcentual en cada sector exportador; cada grupo se encuentra ordenado desde el índice de especialización más bajo hasta el más alto; es decir, desde el grupo de países más diversificado hasta el más concentrado.

\section{Metodología}

La metodología utilizada en el presente estudio es de tipo cuantitativo, con una muestra de 181 países correspondientes al período 1981-2015. Se cuenta con datos de 63 países para los 35 años, 86 países tienen datos disponibles desde 1990 a 2015; y 152 países en los últimos 15 años de la muestra (2000-2015). El estudio tiene alcance descriptivo; las exportaciones de los países están expresadas en dólares y han sido recolectadas de la base de datos de la Organización Mundial de Comercio. Mediante el software estadístico R, se realizó el análisis de conglomerados donde clasificaron a los países cuyos perfiles son muy similares en diversos grupos de acuerdo al índice. Así mismo, con Stata se realizaron estimaciones para establecer cuáles son los factores determinantes de las exportaciones, considerando variables como: PIB, PIB per cápita, Población, Exportaciones en base al PIB, y finalmente, la ubicación geográfica.

\section{Resultados}

Con los datos de las exportaciones procesados y analizados estadísticamente, mediante la elaboración del clúster se tiene que los países que pertenecen al primer grupo del clúster son aquellos que tienen el índice de especialización más bajo (0.072) es decir, su cartera exportable es bastante dinámica en sus economías, los sectores exportadores se encuentran bastante diversificados. En este conjunto de países se destacan principalmente Holanda y Estados Unidos dado que poseen los índices de especialización más bajos a nivel mundial con 0.03 y 0.04 puntos respectivamente, estos países se caracterizan por ser dueños de una desarrollada oferta comercial basadas en: hidrocarburos, reactores nucleares, equipos electrónicos, minerales, productos farmacéuticos, químicos orgánicos, etc.

El grupo de países con la más alta concentración exportadora representa aquellos cuyas economías dependen en gran medida del petróleo, que pertenecen a la Organización de Países Exportadores de Petróleo (OPEP) a excepción de Azerbaiyán y Brunei. Las exportaciones de crudo representan para estos países el $95 \%$ del total, haciendo 
de estas economías sean bastante vulnerables sobre todo a constantes cambios en los precios internacionales de crudo.

Países como Angola, Azerbaiyán, Kuwait, Libia, Venezuela e Irak basan sus actividades en la explotación y refinamiento del petróleo principalmente, concentrando más del $80 \%$ de su PIB en este rubro, trayendo consigo graves consecuencias para sus economías, sobre todo en las crisis petroleras.

Figura 1. Índice de Especialización de exportaciones por países

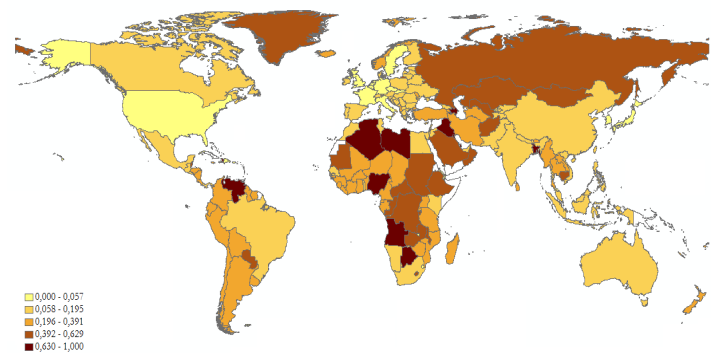

Fuente: Elaborado por los autores a partir de las cifras de las Organización Mundial del Comercio (2017).

3.1 Estimación de los factores determinantes del Índice de especialización de las exportacionesción de los factores determinantes del Índice de especialización de las exportaciones

En el modelo se estimaron las siguientes variables: población, PIB, PIB capital, exportaciones en base al PIB, y área geográfica, planteamos modelos para observar si cada una de las variables es significativa, y si la región juega o no un papel importante en las características de exportación de los países.

\subsubsection{Modelo 1}

Índice Herfindahl $=\beta_{0}+\beta_{1}$ Población $+\epsilon$

\subsubsection{Modelo 2}

Índice Herfindahl $=\beta_{0}+\beta_{1} \mathrm{PIB}+\epsilon$

Los modelos 1 y 2 explican que cuanto mayor es un país, menor el índice. Es lógico pues su economía puede ser más diversa, y, por lo tanto, su actividad exportadora estará enfocada en varios sectores.

\subsubsection{Modelo 3}

Índice Herfindahl $=\beta_{0}+\beta_{1}$ PIB per cápita $+\epsilon$

El modelo 3 muestra que países con mayor PIB per cápita tienen menores índices de especialización. Economías más ricas exportan más variedad de productos mientras que países en desarrollo tienen una mayor concentración. Consistentemente, Papageorgiou \& Spatafora (Papageorgiou y Spatafora, 2013) consideran que mayores ingresos per cápita se asocian ampliamente a una mayor calidad de las exportaciones a nivel de país.

Tabla 2 Resultados de los modelos de panel

\begin{tabular}{|c|c|c|c|c|c|c|c|c|c|c|c|c|}
\hline \multirow{2}{*}{$\begin{array}{c}\text { Variable } \\
\text { Población }\end{array}$} & \multicolumn{2}{|c|}{ modelo 1} & \multicolumn{2}{|c|}{ modelo 2} & \multicolumn{2}{|c|}{ modelo 3} & \multicolumn{2}{|c|}{ modelo 4} & \multicolumn{2}{|c|}{ modelo 5} & \multicolumn{2}{|c|}{ modelo 6} \\
\hline & $-2.70 \mathrm{e}-10$ & $* * *$ & & & & & & & $-2.22 \mathrm{e}-10$ & $* * *$ & $-1.96 \mathrm{e}-10$ & $* * *$ \\
\hline & $2.74 \mathrm{e}-11$ & & & & & & & & $2.93 e-11$ & & $2.68 \mathrm{e}-11$ & \\
\hline \multirow[t]{2}{*}{ PIB } & & & $-4.32 \mathrm{e}-14$ & $* * *$ & & & & & $-2.19 \mathrm{e}-14$ & $* * *$ & $-3.38 \mathrm{e}-15$ & \\
\hline & & & $3.69 \mathrm{e}-15$ & & & & & & $4.15 e-15$ & & $3.61 \mathrm{e}-15$ & \\
\hline \multirow[t]{2}{*}{ PIB PC } & & & & & $-2.39 e-06$ & $* * *$ & & & $-2.51 \mathrm{e}-06$ & $* * *$ & $8.83 e-07$ & $* *$ \\
\hline & & & & & $2.60 \mathrm{e}-07$ & & & & $2.81 \mathrm{e}-07$ & & $2.69 \mathrm{e}-07$ & \\
\hline \multirow[t]{2}{*}{ Exports/pib } & & & & & & & 0.05935 & $* *$ & 0.08301 & $* * *$ & .1054 & $* * *$ \\
\hline & & & & & & & 0.01715 & & 0.01755 & & .01567 & \\
\hline \multirow[t]{2}{*}{ África } & & & & & & & & & & & .3636 & $* * *$ \\
\hline & & & & & & & & & & & .01212 & \\
\hline \multirow[t]{2}{*}{ Asia Oriental } & & & & & & & & & & & .1969 & $* * *$ \\
\hline & & & & & & & & & & & .01452 & \\
\hline \multirow[t]{2}{*}{ Europa } & & & & & & & & & & & .1798 & $* * *$ \\
\hline & & & & & & & & & & & .01298 & \\
\hline \multirow[t]{2}{*}{$\begin{array}{c}\text { América } \\
\text { Latina y el C. }\end{array}$} & & & & & & & & & & & .2973 & $* * *$ \\
\hline & & & & & & & & & & & .01163 & \\
\hline \multirow[t]{2}{*}{ MENA } & & & & & & & & & & & .4533 & $* * *$ \\
\hline & & & & & & & & & & & .01238 & \\
\hline \multirow[t]{2}{*}{ Asia del Sur } & & & & & & & & & & & .2657 & $* * *$ \\
\hline & & & & & & & & & & & .01734 & \\
\hline \multirow[t]{2}{*}{ Constante } & .3532 & $* * *$ & .3573 & $* * *$ & .3636 & $* * *$ & .3272 & $* * *$ & .35247 & $* * *$ & & $* * *$ \\
\hline & .005715 & & .008095 & & .005304 & & .0071 & & .006273 & & & \\
\hline
\end{tabular}

Elaborado por: Autores 


\subsubsection{Modelo 4}

Índice Herfindahl $=\beta_{0}+\beta_{1}$ export $/ \mathrm{PIB}+\epsilon$

El modelo 4 afirma que una mayor importancia de las exportaciones para la economía del país, medida como el porcentaje de exportaciones respecto al PIB, se relaciona con un índice más alto. Esto indicaría que países más especializados tienden a tener mayor nivel de exportaciones que países con el mismo nivel de producción, pero con exportaciones menos concentradas. Es decir, países muy especializados, como los petroleros, exportan una mayor porción de su PIB que otros con exportaciones más diversas.

\subsubsection{Modelo 5}

Índice Herfindahl $=\beta_{0}+\beta_{1}$ Población $+\beta_{2} \mathrm{PIB}+\beta_{3}$ PIB per cápita $+\beta_{4}$ Export/PIB $+\epsilon$

En el modelo 5 incluyen todas las variables que no se detectan problemas de correlación entre ellas. Todas son significativas y se mantienen las conclusiones de los cuatro modelos previos.

\subsubsection{Modelo 6}

Índice Herfindahl $=\beta_{0}+\beta_{1}$ Población $+\beta_{2}$ PIB $+\beta_{3}$ PIB per cápita $+\beta_{4}$ Exportación $/ \mathrm{PIB}+\beta_{5}$ África $+\beta_{6}$ Asia Oriental $+\beta_{7}$ Europa y Asia Central, $\beta_{8}$ América Latina y el Caribe $+\beta_{9}$ MENA $+\beta_{1} 0$ Asia del Sur $+\epsilon$

En el último modelo se incorporan las regiones bajo la definición del Banco Mundial: África, Asia Oriental, Europa y Asia Central, América Latina y el Caribe, Oriente Medio y África del Norte (MENA), Asia del Sur, y Desarrollados que se omite en el modelo. Todas las variables conservan su significatividad menos el PIB. Una vez incorporado el efecto de la región un mayor PIB per cápita contribuye a la especialización, indicando que, aunque a nivel global los países con mayor renta son aquellos menos especializados, a nivel regional, los países con mayor renta son los que presentan la mayor concentración de las exportaciones.

Todas las regiones incluidas en el modelo presentan valores más altos para el HHI que los países desarrollados, una vez se tiene en cuenta el efecto del resto de variables. La región con un efecto más elevado es MENA, seguida de África. En un tercer lugar se encontrarían América Latina y el Caribe y Asia del Sur, y por último Asia Oriental y el resto de Europa y Asia Central. La explicación de este hecho se encuentra principalmente en la gran presencia de materias primas en algunas de estas regiones.

El $r^{2}$ experimenta un gran ascenso hasta 0.3128 por lo que el mayor determinante de especialización de las exportaciones debe ser buscado en la geografía que condiciona la ventaja comparativa de los países, así como la disponibilidad de las materias primas. Es decir, la mayor parte de la explicación del modelo se encuentra en la variable del área geográfica, no hay una relación de causalidad, pero se muestra una relación positiva con la variable dependiente.

\section{Conclusiones y Recomendaciones}

En esta investigación se ha determinado que la diversificación exportadora genera que los países puedan responder más eficientemente ante fluctuaciones en los precios internacionales. De hecho, en este mismo sentido, Sannassee et al. (2014) sugiere que la causa principal de la inestabilidad de los ingresos por exportaciones es la concentración de las exportaciones en determinados productos básicos y mercados. En el caso de países como la Holanda, Estados Unidos o la República de Corea, que no solo exportan miles de millones de dólares al año, sino que ofrecen a nivel mundial, productos de diversos sectores y actividades económicas, teniendo los índices de especialización más bajos de todos los países de la muestra.

Por el contrario, los países con el Índice Herfindahl más alto son Irak, Groenlandia y Angola, dado que concentran sus exportaciones en más del $95 \%$ en un solo sector; los cuales se enfrentan con serios obstáculos que frenan el comercio y la diversificación, tales pueden ser infraestructura insuficiente, débil gestión institucional, ineficientes sistemas de transporte, ubicación geográfica, barreras paraarancelarias y administrativas.

Esa débil competitividad comercial puede ser contrarrestada con ayuda de los gobiernos nacionales mediante el diseño de políticas que incentiven la producción de bienes y servicios con valor agregado y promover el acceso a tecnologías modernas, además de una controlada gestión de las variables económicas como la inflación, desempleo, tipos de cambio y de protección de inversiones; efectuar políticas en torno a medidas redistributivas de la riqueza, acumulación de capital humano, mejoras en infraestructura, fomentando la investigación y desarrollo. Hay que reconocer que un proceso de diversificación es difícil y toma su tiempo, pero los beneficios que se generan son significativos.

Además, un mayor PIB per cápita genera mayor especialización a nivel regional, pero a nivel global los países con mayores ingresos son más diversificados. Consistentemente, Agosin (2009) señala que existe una relación de causalidad entre el ingreso per cápita y la diversificación de las exportaciones, claro está no es el único, pero sin duda es un indicador de competitividad internacional. Por otro lado, Sannassee et al. (2014) argumenta que los países menos desarrollados son los menos diversificados y, por lo tanto, los que tienen una frontera de diversificación más cerrada que los países avanzados.

Países con ingresos más bajos y con grandes distancias geográficas hacia los principales mercados tienen escasa oportunidad para diversificar por los altos costes logísticos y de transporte que se debe asumir. Y los países más grandes son más diversos, pero al incluir la variable de área geográfica el PIB pierde significancia, pero el coeficiente de determinación aumenta, por lo tanto, a nivel global los países con mayor renta son menos especializados, a nivel regional los países más ricos son los que presentan niveles altos de concentración de las exportaciones. 


\section{Referencias Bibliográficas}

Agosin, M. (2009). Crecimiento y diversificación de las exportaciones en economías emergentes. Revista Cepal, 97, 117-133.

Carrère, C., Cadot, O., y Strauss-Kahn, V. (2011). Trade diversification: Drivers and impacts. in: Jansen, $m$. \& peters, $r . \&$ salazar-xirinachs, j.-m. trade and employment: from myths to facts.

Chenery, H. B., Robinson, S., y Syrquin, M. (1986). Industrialization and growth: a comparative study. Washington: World Bank.

Cosh, A. (1987). The new palgrave: A dictionary of economics. En (cap. Diversification of activities). Palgrave Macmillan.

Expósito, A. (2003). Especialización, convergencia y concentración de las exportaciones españolas de mercancías. un análisis regional (1991-2001). Boletín económico de ICE, 27-36.

Hausmann, R., y Klinger, B. (2006). Structural transformation and patterns of comparative advantage in the product space. Cambridge, MA, Center for International Development at Harvard University.

Hesse, H. (2009). Export diversification and economic growth. breaking into new markets: emerging lessons for export diversification.

Pacek, N., y Thorniley. (2008). Oportunidades en los mercados emergentes. Buenos Aires: Cuatro media.

Papageorgiou, C., y Spatafora, N. (2013). Economic diversification in low-income countries: stylized facts. IMF Note.
Recibido: 30 de junio de 2018

Aceptado: 3 de septiembre de 2018

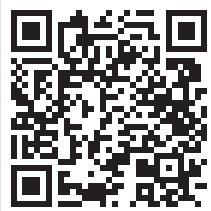

